PDFlib PLOP: PDF Linearization, Optimization, Protection

Page inserted by evaluation version www.pdflib.com - sales@pdflib.com 


\title{
GRAPHICAL CONFLICT I: A NEW CLASS OF EXTREMAL PROBLEMS
}

\author{
Frank Harary and Jerald A. Kabell \\ Department of Mathematics \\ The University of Michigan \\ Ann Arbor, Michigan 48109
}

\section{INTRODUCTION}

In the psychological literature, there are traditionally three types of conflict: approach/approach, approach/avoid, and avoid/avoid (see e.g., [2]). The first of these, approach/approach, involves two very desirable alternatives $a_{1}$ and $a_{2}$ such that the subject cannot fully attain both of them. (In fairy tales the handsome young prince is having trouble deciding whether to take the ugly, but rich, princess or the beautiful servant girl to the ball.) The second case entails a goal $a_{1}$ which is sought and some evil, horrible alternative $a_{2}$ which the subject wishes to avoid while approaching $a_{1}$, but must at least partially confront in order to do so. (The prince must slay the dragon before he can try to woo the princess.) Finally, the avoid/avoid conflict forces the subject to choose one of two evils, hopefully the lesser. (Most presidential elections come to mind.)

In graph theoretic terms a similar sort of tension can be created by attempting to achieve simultaneous extreme values of two different parameters. It is natural in this context to identify the approach to a goal with the maximization of a parameter, while avoidance becomes minimization. The questions which suggest themselves are:

1. What are the simultaneous extreme values of the parameters?

2. What are the extremal graphs attaining these values?

3. When is there a unique extremal graph?

\section{DEFINITIONS AND Notation}

We begin by making precise the notion of the simultaneous extremum of two different graphical parameters $a_{1}$ and $a_{2}$. It is clearly insufficient to combine the individual extrema, as, in general, they may be expected to occur under different conditions; hence we are led to consider various possible expressions combining the two. The simplest of these are of course $a_{1}+a_{2}, a_{1} a_{2}, a_{1}-a_{2}$ and $a_{1} / a_{2}$. The first two suggest themselves naturally in those cases where the desired extrema are of the same type, i.e., both maxima or both minima. While both possibilities have much to recommend them, the second is more sensitive to variations of the smaller parameter. Therefore we choose to define the simultaneous maximum (or minimum) of $a_{1}$ and $a_{2}$ as the maximum (or minimum) of their product. Note that this is also in accordance with the ancient dictum: "Go forth and multiply." When the extrema desired are of opposite types (one maximum, one minimum) we are naturally drawn to consider either the difference or the quotient. By the same rationale, we opt for the quotient, with the parameter to be maximized appearing as the numerator, that is, we shall take the simultaneous maximum of $a_{1}$ and the minimum of $a_{2}$ to be the maximum of the quotient $a_{1} / a_{2}$. Bowing, however, to the XIth Commandment, "Thou shalt not divide by zero!," we require $a_{2} \neq 0$. 
Henceforth we will use the following notation to indicate various types of extremal problems. Given a parameter $a_{1}$ and constraints $c_{1}, c_{2}, \ldots, c_{n}$, we denote the maximum (or minimum) of $a_{1}$ subject to $n$ given constraints $c_{i}$ by $\max \left(a_{1}: c_{1}, c_{2}, \ldots, c_{n}\right)$ or min $\left(a_{1}: c_{1}, c_{2}, \ldots, c_{n}\right)$. For the simultaneous maximum of $a_{1}$ and $a_{2}$ we write $\max \left(a_{1} a_{2}: c_{1}, c_{2}, \ldots, c_{n}\right)$ and similarly for the simultaneous minimum. Finally, for the maximum of $a_{1}$ and minimum of $a_{2}$, we consider $\max \left(a_{1} / a_{2}: c_{1}, c_{2}, \ldots, c_{n}\right)$. One additional piece of notation which we find useful in describing some of the extremal graphs is $K(p / t)$ for the equipartite graph dividing $p$ points into $t$ parts. Thus this is the complete $t$-partite graph in which each part has either $\{p / t\}$ or $[p / t]$ points. All other definitions and notations follow [5].

\section{Point- ANd Line-Independence AND Covering}

We begin by considering four related parameters - the point and line covering numbers $\left(\alpha_{0}\right.$ and $\left.\alpha_{1}\right)$ and the point and line independence numbers $\left(\beta_{0}\right.$ and $\left.\beta_{1}\right)$. Obviously $\max \left(\beta_{0}: p\right)=p$ and $\max \left(\beta_{1}: p\right)=[p / 2]$. As a first result we establish the following analog of an approach/approach conflict.

THEOREM 1. $\max \left(\beta_{0} \beta_{1}: p\right)=\left[p^{2} / 4\right]$.

Proof: If we begin with the complete graph $K_{p}$ and select any maximal independent set of lines $L$, we have $\beta_{1}=[p / 2]$ and $\beta_{0}=1$. It is clear that deleting any line not in $L$ will not affect $\beta_{1}$ and will not decrease $\beta_{0}$. Thus if we remove all the lines other than those of $L$ we obtain the graph $G=[p / 2] K_{2} \cup(\{p / 2\}-[p / 2]) K_{1}$, for which $\beta_{1}=[p / 2]$ and $\beta_{0}=\{p / 2\}$, giving $\beta_{0} \beta_{1}=\left[p^{2} / 4\right]$. To see that this is indeed the maximum possible value, we need only observe that $\beta_{0} \leq p-\beta_{1}$ and therefore $\beta_{0} \beta_{1} \leq \beta_{0}\left(p-\beta_{0}\right)$.

Corollary 1a. For any extremal graph with maximum $\beta_{0} \beta_{1}, \beta_{0}=\{p / 2\}$ and $\beta_{1}=[p / 2]$.

The graph $G$ we have constructed here is not unique; the path $P_{p}$ has precisely the same value of $\beta_{0}$ and $\beta_{1}$, as does the cycle $C_{p}$, for even $p$. In fact, if we selected a maximal independent set of points $V_{0}$ in $G$, any graph constructed from $G$ by adding any collection of lines none of which joins two points of $V_{0}$, will be an extremal graph, and, in view of COROLLARY la, every extremal graph may be constructed in this way. Thus it is clear that in the class of extremal graphs, the unique graph for which $q$ is also minimized is $G$.

Since the independence numbers $\beta_{0}$ and $\beta_{1}$ and the covering number $\alpha_{0}$ and $\alpha_{1}$ are related by the well-known result of Gallai [3] that $\alpha_{0}+\beta_{0}=\alpha_{1}+\beta_{1}=p$, it might be expected that THEOREM 1 would lead to a number of immediate corollaries relating them in pairs. This is not the case, however! We note, of course, that $\max \left(\alpha_{0} \beta_{0}: p\right)=\max \left(\alpha_{1} \beta_{1}: p\right)=\left\{\left(p^{2}-1\right) / 4\right\} \quad$ (both realized by $\left.C_{p}\right)$ and $\min \left(\alpha_{0} \beta_{0}: p\right)=\min \left(\alpha_{1} \beta_{1}: p\right)=p-1$ (with extremal graphs $K_{2} \cup \bar{K}_{p-2}$ and $K_{1, p-1}$, respectively), but these follow directly from Gallai's result, independently of THEOREM 1. The other combinations prove not so tractable. In the case of $\alpha_{0} \alpha_{1}$, for example, the complete graph $K_{p}$ yields a value of $\left(p^{2}-p\right) / 2$ if $p$ is even or $\left(p^{2}-1\right) / 2$ if $p$ is odd, and the star $K_{1, p-1}$ gives $\alpha_{0} \alpha_{1}=p-1$.

We originally conjectured these to be the maximum and minimum. At this interesting and stimulating conference, Chung, Erdös, and Graham [1] showed that the minimum is indeed given uniquely by $K_{1, p-1}$ and, when $p$ is odd, the maximum by $K_{p}$. When $p$ is even, they proved that $\max \left(\alpha_{0} \alpha_{1}: p\right)=\left(p^{2}-4\right) / 2$ with extremal 
graph $K_{4}$ if $p=4$ and $K, \cup K_{s}$ with $r, s>1$, odd, and $r+s=p$ when $p>4$. The original conjecture is true however if the graph is additionally required to be connected.

\section{CONNECTIVITY AND COLORING}

The concepts of connectivity and colorability of a graph provide us with two further groups of related invariants, whose mutual interaction is rather interesting. As an example we consider first the (point) connectivity $\kappa$ and the (point) chromatic number $\chi$.

THEOREM 2. $\max (\kappa / \chi: p)=[p / 2] / 2$.

Proof: For a given value of $\chi$, it is evident that the maximum connectivity (hence also the maximum value of $\kappa / \chi$ ) will occur in the case of the equipartite graph $K(p / \chi)$. This gives $\kappa=p-\{p / \chi\}$, or $\kappa / \chi=(p-\{p / \chi\}) / \chi$. It is readily established that this expression is decreasing for $\chi \geq 2$, hence its maximum occurs at $\chi=2$. It is also clear that the unique extremal graph in this case is $K(p / 2)=K([p / 2]$, $\{p / 2\})$.

Looking at the same problem in the opposite direction, we see immediately that $\max \{\chi j \kappa: p\}=p-1$ (with the unique extremal graph $K_{p-1} \cdot K_{2}$ in the notation of $[5, p .23]$ ). In addition we should note that $\max \{\kappa \chi: p\}=p^{2}-p$ (this is immediately clear if we consider $K_{p}$ ), and $\min \{\kappa \chi: p, G$ connected $\}=2$ (the path $P_{p}$ is one of many possible graphs realizing this limit). In each of the cases the proofs proceed identically if we replace $\kappa$ by $\lambda$ wherever it occurs.

\section{Covering AND CONNECTIVITY}

As an example of the type of interaction which may be expected between parameters of these families, we consider $\kappa$ and $\beta_{0}$.

THEOREM 3. $\max \left(\kappa \beta_{0}: p\right)=\left[p^{2} / 4\right]$.

Proof : Since any member of a set of independent points is adjacent to at least $\delta$ points (which therefore cannot belong to that set), we see that $\beta_{0} \leq p-\delta$. But it is well known that $\kappa \leq \delta$, so that $\kappa \beta_{0} \leq \delta(p-\delta)$; hence $\kappa \beta_{0} \leq\left[p^{2} / 4\right]$. To verify that this bound can actually be attained, consider again the graph $K([p / 2],\{p / 2\})$.

It is clear, of course, that $\min \left(\kappa \beta_{0}: p\right)=2$ (simply consider the graph $K_{r} \cdot K_{s}$, where $r, s>0$ and $r+s=p+1)$.

One is immediately struck by the fact that both the bound and the extremal graph of THEOREM 3 are the same as those of Turán's theorem [7], but we hasten to point out that the extremal graph is not unique. In fact, any graph $G=H+\bar{K}_{(p / 2)}$, where $H$ has $[p / 2]$ points, will serve equally well.

\section{UnSOlved Problems}

Clearly we have been able to address only a minute proportion of the potential questions in this fertile new field. Our object here is to open this fascinating new topic within extremal graph theory, not to exhaust it. Many other readily accessible results could be mentioned here, e.g., $\min \left(\chi \beta_{0}: p\right)=p\left(\bar{K}_{\mathrm{p}}\right.$ is one of many graphs 
realizing this bound) and $\max \left(\chi^{\prime} / \chi: p\right)=(p-1) / 2$ (again the star $K_{1, p-1}$ is an extremal example).

Also deserving of mention are some genuinely formidable problems. Foremost among these, perhaps, are those involving the various topological invariants, such as $\max (\gamma / v: p)$, or $\max (\theta / \xi: p)$, where, as usual, the notation $\gamma, v, \theta, \xi$ stand for the genus, crossing number, thickness, and coarseness of a graph, respectively. For the products, it appears certain that $\max (\gamma v: p)$ and $\max (\theta \xi: p)$ will be realized by $K_{p}$, and we conjecture that this can be proved without having to explicitly determine $v\left(K_{p}\right)$ or $\xi\left(K_{p}\right)$, which have proved to be very difficult problems indeed. Some work has been done which indicates a possibility of success in this area (see, for example, the result of Harary et al. [6] that there exist graphs of genus 1 with arbitrarily high crossing number), but this has, in general, dealt only with basically unconstrained situations. There are also the previously mentioned combinations of covering and independence numbers, although these are probably not of the same order of magnitude of difficulty. Beyond these, both [4] and the Index of Symbols in [5] give a wide selection of graphical parameters, any pair of which may be considered in each of the four ways we have indicated.

It might also be interesting to investigate the combinations of two graphical parameters under the sum and difference operations. Of course, it becomes much more complex, but sometimes feasible to consider both $p$ and $q$ as constraints. One can also propose other conditions such as prescribed girth, diameter, connectivity, and so forth. Another variation on these problems would be analogous to a conflict situation where more than two positive or negative alternatives are involved. Of course, this is far more complex than the two-alternative situation, but it appears likely that some of the resulting problems will be tractable. We plan to investigate several of these variations in future communications in this series.

\section{SummaRY}

Inspired by the analogy with the three traditional types of conflict in psychology (approach/approach, approach/avoid, and avoid/avoid), we consider corresponding types of extremal problems in graph theory. These are translated into the determination of extremal values of the product or quotient of two graphical parameters, subject to given constraints. For purposes of exemplification, we study and determine both the exact solution and the extremal graphs for several products and quotients which include confrontations of (a) point- and line-independence and covering, (b) connectivity and coloring, (c) covering and connectivity. We conclude with an indication of several unsolved problems for future research.

\section{REFERENCES}

1. Chung, F. R. K., P. Erdös \& R. L. Graham. On the product of the point and line covering numbers of a graph. In this volume. pp. 597.

2. CoOmBS, C. L.\& G. S. AvRUNIN. 1977. Single-peaked functions and the theory of preference. Psychol. Rev. 84: 216-230.

3. Gallai, T. 1959. Uber extreme Punkt- und Kantenmengen. Ann. Univ. Sci. Budapest, Eötvös Sect. Math. 2: 133-138.

4. Harary, F. 1969. The Greek alphabet of graph theory. In Recent Progress in Combinatorics. W. T. Tutte, Ed. Academic Press, New York. pp. 13-20.

5. Harary. F. 1969. Graph Theory. Addison-Wesley, Reading Mass. 
6. Harary, F., P. C. Kainen, \& A. J. Schwenk. 1973. Toroidal graphs with arbitrarily high crossing numbers. Nanta Math. 6: 58-67.

7. Turán, P. 1941. Eine Extremalaufgabe aus der Graphentheorie. Mat. Fiz. Lapok (in Hungarian). 48: 436-452. 